\title{
To Improve the Recognition Rate with High Security with Ear/Iris Biometric Recognition Technique with feature Extraction \& Matching
}

\author{
Paridhi Agarwal ${ }^{1}$, Dr. Naveen Hemrajani ${ }^{2}$ \\ ${ }^{12}$ (Computer Science and Engineering Department, Suresh Gyan Vihar University, Jagatpura, Jaipur, India)
}

\begin{abstract}
Ears are the new biometric with major advantage in that they appear to maintain their structure with increasing age. Contending that the ear is mainly a planar shape $2 D$ images are used, which are consistent with deployment in surveillance and other planar-image scenarios. In this paper, a new model-based approach to ear biometrics is proposed. The model parts are derived by a stochastic clustering method on a set of scale invariant features on a training set. The model description is extended by proposing a new wavelet-based analysis with a specific aim of capturing information in the ear's outer structures. The performance is evaluated on test sets selected from the XM2VTS database. By results, both in modeling and recognition, the new modelbased approach does indeed appear to be a promising new approach to ear biometrics. The recognition performance has improved notably by the incorporation of the new wavelet-based analysis. A model-based approach has an advantage in handling noise and occlusion. A wavelet can offer performance advantages when handling occluded data by localization. A robust matching technique is also added to restrict the influence of corrupted wavelet projections. Furthermore, the automatic enrolment is tolerant of occlusion in ear samples. The hybrid method obtains promising results recognizing occluded ears. The results have confirmed the validity of this approach both in modeling and recognition. The new hybrid method does indeed appear to be a promising new approach to ear biometrics, by guiding a model-based analysis via anatomical knowledge.
\end{abstract}

Keywords: hybrid method, model-based approach, stochastic clustering, XM2VTS database, wavelet-based analysis

Submitted date 27 June 2013

Accepted Date: 02 July 2013

\section{Introduction}

Biometrics is the science in which an entity is distinguished on the basis of physiological features or behavioral characteristics [1]. Physiological characteristics include finger print, iris scan, retina scan, face, thermo grams of face, palm print, ear etc. whereas behavioral characteristics consist of gait recognition, odour, voice recognition and signature verification. The results are obtained in biometrics by using single or multiple means. The achieved results indicate that biometric techniques are much more precise and accurate than the traditional techniques. Other than precision, there have always been certain problems which remain associated with the existing traditional techniques. As an example consider possession and knowledge. Both can be shared, stolen, forgotten, duplicated, misplaced or taken away. However the danger is minimized in case of biometric means [2]. In this paper, I have evaluated and improved the Recognition Rate with High Security with Ear/Iris Biometric Recognition Technique with feature Extraction \& Matching.

\section{Biometrics Architecture}

Common Criteria Biometric Evaluation Methodology Working Group in their report Biometric Evaluation Methodology published in 2002 divides a biometric system into four main components, as described in the figure [1]. 
User

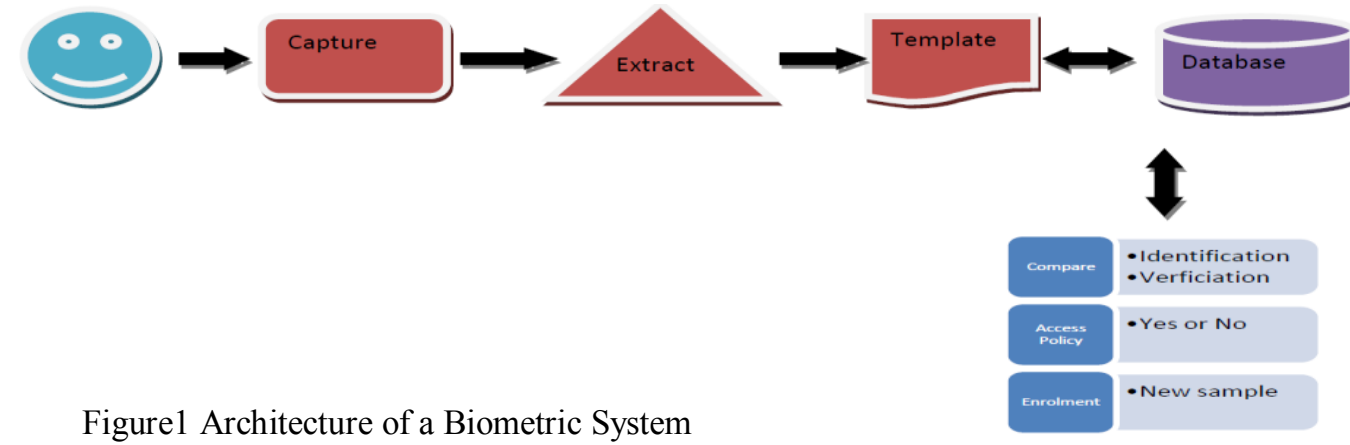

III. Biometric Through An Eye

Biometric verification can be done through an eye which is considered as the most reliable authentication procedure to date, almost with zero false accepting rates. It is relatively new technology as compared to the other leading biometric techniques such as finger printing. Authentication in an eye can be done through two means, that is, either iris or retina [4].

US Biometric.gov explains that before the image capture, localization of the landmark sights of an eye should be made carefully by the system because the quality of the captured image extraction depends upon it [5]. These landmark features allows the system to isolate the iris portion of an eye while capturing the image and later extract the desired portion [5]. As per the Figure, localization of the eyelids boundaries and the iris is marked by a white line.

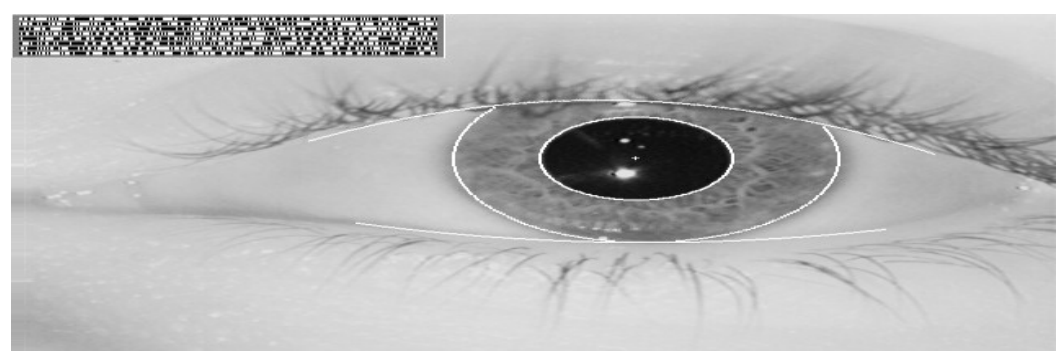

\subsection{Far And Frr Table}

Iris Recognition Technology for Improved Authentication published at SANS Institute Reading Room site that False Accepting Rate and False Rejecting Rate of Iris Recognition is:

Table 1. Hamming Distances and Error Probabilities

\begin{tabular}{|l|l|l|}
\hline Hamming Distance & False Accept Probability & False Reject Probability \\
\hline 0.28 & 1 In 1012 & 1 in 11,400 \\
\hline 0.29 & 1 In 1011 & 1 in 22,700 \\
\hline 0.30 & 1 in 6.2 billion & 1 in 46,000 \\
\hline 0.31 & 1 In 665 million & 1 in 95,000 \\
\hline 0.32 & 1 In 81 million & 1 in 201,000 \\
\hline 0.35 & 1 In 295,000 & 1 in 2.12 million \\
\hline 0.37 & 1 In 12300 & 1 in 11.3 million \\
\hline
\end{tabular}

\section{Ear Biometrics}

Ear is a relatively new class of biometrics. Ear features have been used for many years in forensic sciences for recognition. Ear is a stable biometric and does not vary with age. Ear has the properties that a biometric trait should possess, i.e. permanence, uniqueness, universality and collectability.

\subsubsection{Voronoi Diagrams}

\section{Methodology For Ear Bio-Metric Systems}

The sample ear image with the corresponding Voronoi diagram and neighborhood graph is presented in Figure . 

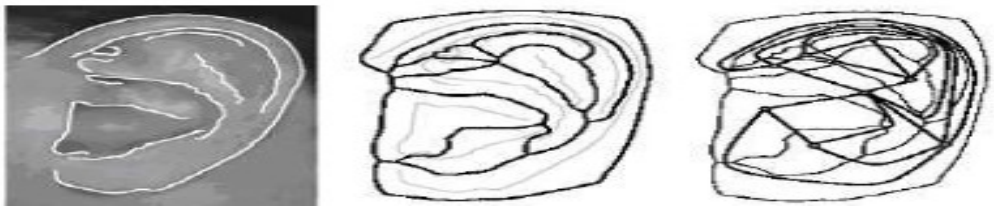

Figure 2 shows Sample ear image with the constructed Voronoi diagram and the corresponding neighborhood

The neighbour of pixel $p$ is pixel q such that graph

$$
\begin{aligned}
& d(p, q)=1 . \text { If } p=(x p, y p) \text { and } q=(x q, y q) \text {, then: } \\
& d(p, q)=\max (|x p-x q|,|y p-y q|)(2)
\end{aligned}
$$

Assuming that we have a contour given as an ordered set of points in the image plane $\mathrm{C}=\{\mathrm{ti} \mid \mathrm{i}=1 \ldots \mathrm{n}\}, \mathrm{ti}=(\mathrm{xi}, \mathrm{yi})$. The contour is usually an open curve.

\subsubsection{Concentric Circle Based Method - Ccm}

The CCM method is based on concentric circles centered in the obtained centroid point

$$
\mathrm{O}=\left\lfloor\frac{r l \max }{\Delta r}\right\rfloor
$$

For $\mathrm{rl}=1 * \Delta \mathrm{r},(1=1, \ldots ., \mathrm{o})$, basing on the record $\mathrm{T}$ we store the number of elements 11 , for which $\mathrm{Rl}=\mathrm{p}$ and we create the vectors:

$$
\begin{aligned}
& \mathrm{Vl}=\left\{\left(1_{1}\right) 1 \ldots\left(1_{1}\right) \mathrm{s} \ldots,\left(1_{1}\right) \mathrm{o}\right\}, \\
& \mathrm{VZ}=\{(\mathrm{i} 1, \mathrm{j} 1, \rho, \varphi 1), \ldots,(\mathrm{is}, \mathrm{js}, \rho, \varphi \mathrm{s}), \ldots,(\mathrm{iu}, \mathrm{ju}, \rho, \varphi \mathrm{u})\}
\end{aligned}
$$

The feature vector that is created on the basis of presented feature extraction algorithm can be formulated as:

$$
\mathrm{V}=\{\mathrm{V} 1, \mathrm{D} 1, \ldots, \mathrm{Vl}, \mathrm{Dl} 1, \ldots, \mathrm{Vo}, \mathrm{Do}\}
$$

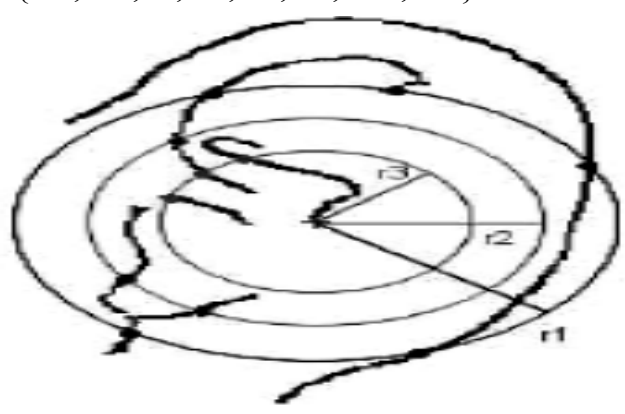

Figure 3 shown the Symbolic representation of algorithm for $\mathrm{Nr}=3$

\subsubsection{Contour Tracking Method - Ctm}

Let go, denote the current point of the contour line, so that $g o=g(i, j)=1$. Contour line points are classified on the basis of the coefficient $\mathrm{Nc} 8$

Where $S=(1,3,5,7)$

$$
\mathrm{Nc} 8=\sum_{k=s}(g k-g k g k+1 g k+2)
$$
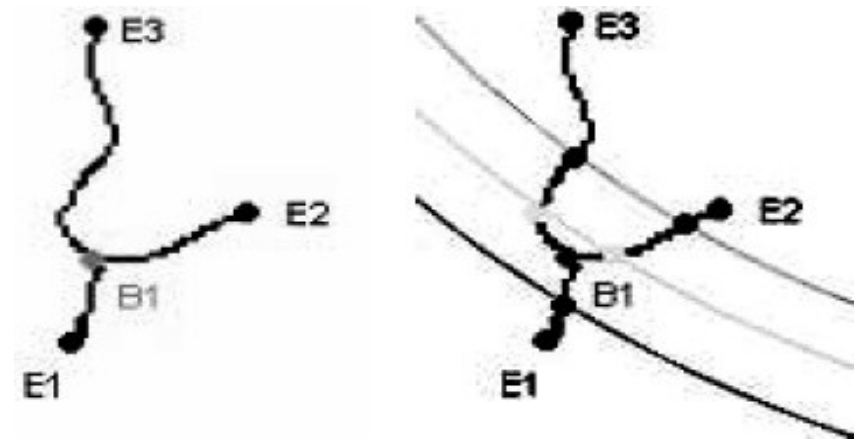

Figure 4. Symbolic presentation of the points extracted by tracing contour method, Ending points $\mathrm{E}$ and bifurcations B are marked in the contour, as well as the intersection points with the consecutive radii rl (right). 
For $\mathrm{C}$ contour in the image we obtain:

$$
F=\left\{\begin{array}{l}
{\left[\begin{array}{l}
\left(l_{t}, N_{E}, N_{B}\right)\left(\left(i_{1}, j_{1}, \rho_{1}\right) \ldots\left(i_{s}, j_{s}, \rho_{s}\right) \ldots\right. \\
\left.\left(i_{u}, j_{u}, \rho_{u}\right)\right)_{r_{l}=1} \ldots\left(\left(i_{1}, j_{1}, \rho_{1}\right) \ldots\left(i_{s}, j_{s}, \rho_{s}\right) \ldots\right. \\
\left.\left(i_{u}, j_{u}, \rho_{u}\right)\right)_{r_{l}=o}\left(\left(i_{1}, j_{1}\right)_{N_{E}=1} \ldots\left(i_{1}, j_{1}\right)_{N_{E}}\right) \\
\left(\left(\left(i_{1}, j_{1}\right)_{N_{B}=1} \ldots\left(i_{1}, j_{1}\right)_{N_{B}}\right)\right.
\end{array}\right]_{c=1}} \\
{\left[\begin{array}{l}
\left(l_{t}, N_{E}, N_{B}\right)\left(\left(i_{1}, j_{1}, \rho_{1}\right) \ldots\left(i_{s}, j_{s}, \rho_{s}\right) \ldots\right. \\
\left.\left(i_{u}, j_{u}, \rho_{u}\right)\right)_{r_{l}=1} \ldots\left(\left(i_{1}, j_{1}, \rho_{1}\right) \ldots\left(i_{s}, j_{s}, \rho_{s}\right) \ldots\right. \\
\left.\left(i_{u}, j_{u}, \rho_{u}\right)\right)_{r_{l}=o}\left(\left(i_{1}, j_{1}\right)_{N_{E}=1} \ldots\left(i_{1}, j_{1}\right)_{N_{E}}\right) \\
\left(\left(\left(i_{1}, j_{1}\right)_{N_{B}=1} \ldots\left(i_{1}, j_{1}\right)_{N_{B}}\right)\right.
\end{array}\right]_{c=C}}
\end{array}\right\}
$$

\subsubsection{Angle - Based Contour Representation Method Abm}

Each extracted contour is treated as an independent open curve. Each contour is represented by two sets of angles [3]:

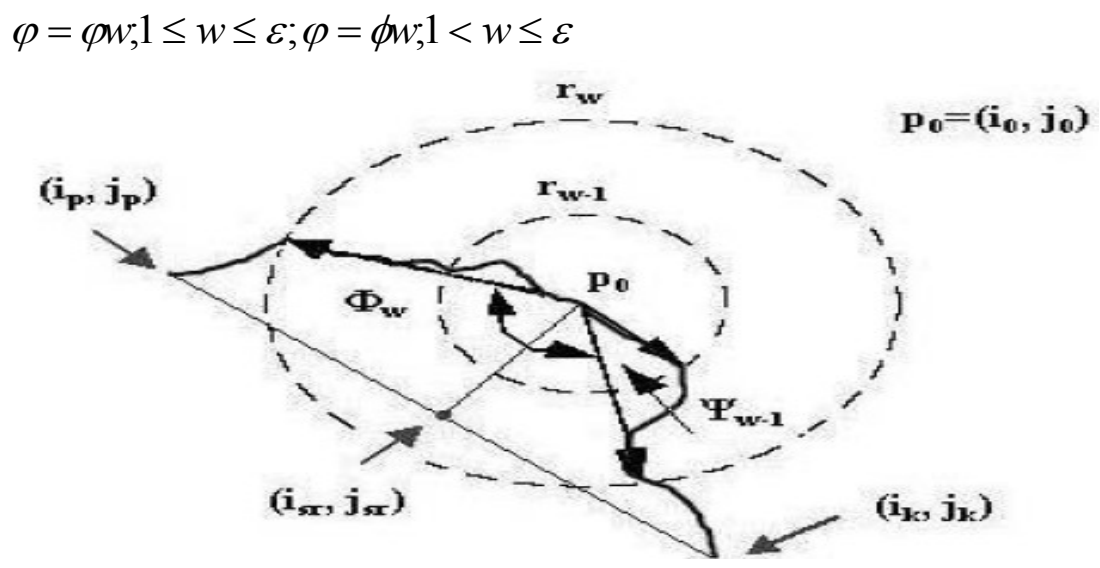

Figure 5. shown the Visualization of the ABM method for a chosen ear contour and 2 radii (concentric circles) with a centre in $\mathrm{p} 0$

Having assumed that there are $\mathrm{C}$ contours in the ear contour image, and that each contour is analyzed by concentric circles, the feature vector is given by:

$$
\mathrm{W}=\left\{\left(\Phi \mathrm{w}, \Psi_{\mathrm{w}}-1\right) 1, \ldots,\left(\Phi \mathrm{w}, \Psi_{\mathrm{w}}-1\right) \mathrm{c}, \ldots,(\Phi \mathrm{w}-1) \mathrm{c}\right\}
$$

\subsubsection{Geometrical Parameters Method - Gpm}

Extraction of the longest contour Lemax within the ear image, contour length is calculated according to:

Where:

Q- Number of contour c- Number of contours, for

$$
L_{c}=\sum_{q=1}^{Q-1} \sqrt{\left(x_{q+1}-x_{q}\right)^{2}+\left(y_{q+1}-y_{q}\right)^{2}} \begin{aligned}
& \text { points; } \\
& \mathrm{c}=1 \ldots \mathrm{C} ;
\end{aligned}
$$

$(\mathrm{x}, \mathrm{y})$ - coordinates of contour points;

$\mathrm{q}$ - Indexation of the current contour point.

The feature vector computed by the proposed method GPM containing the parameters is given by:

$$
\mathrm{FV}=\{(\mathrm{Lc}, \mathrm{dkp}, \mathrm{kk}, \mathrm{d}, \mathrm{b}, \mathrm{db}, \mathrm{cc}) \mathrm{c}\}
$$

\subsubsection{Proposed Algorithm:-}

\section{A. Algorithm for Database}

Step 1: Declaration of the Empty Vector in accordance with the size of image data

Step 2: Reshape the matrix into the vector whose elements are collected from the various images.

Step 3: Provide the Space for each separate image into the dataset

\section{B. Algorithm for Recognition}

Step 1: Mean and variance of the collected iris/ear images are made almost equal using mean and variance normalization technique. Mean and variance of one of the image from the collections is treated as desired mean and desired variance. 
Step 2: Reshape the matrix into the vector whose elements are collected column by column from the matrix.

Step 3: Co-variance matrix of the collected vectors is computed. Eigen values of the co-variance matrix and the Eigen vectors corresponding to the significant Eigen vectors are computed

Step 4: Eigen vectors are reshaped into the matrix of the original size. They are called Eigen iris/ ears.

Step 5: The Eigen iris/ears are orthogonal to each other. They can be made orthonormal to each other by normalizing the vectors.

Step 6: For every iris/ear image matrix, feature vectors are obtained as the inner product of eigen basis vectors and the reshaped iris/ear image matrix.

Step 7: Mean vector of the feature vectors collected from the same person is treated as the template assigned to that corresponding person. This is repeated for other persons also. Thus one template is assigned to every person and they are stored in the database.

Step 8: To classify the unknown iris/ear image as one among the four categories, template is computed as the inner product of Eigen basis vectors (Eigen iris/ ears) with reshaped normalized unknown ear image. The template thus obtained is compared with the group of templates stored in the database using Euclidean distance.

Step 9: Template corresponding to the minimum Euclidean distance is selected and the person corresponding to that template is declared as the identified person.

\section{Tools Used}

For the analysis of the proposed method MATLAB on a PC with $2.0 \mathrm{GHz}$ Dual Core Intel processor and 2GB RAM is to be used. The MATLAB is widely used scientific tool. This provide wide tool for the scientific and research tools to the user for simulation and for real time data processing. The MATLAB scripting is user friendly and easy to debug tool, and ideal for the scientific, complex modeling.

\section{Results}

Hamming Distance Vector Method is used for Identification. In this method we have used statistical analysis for Distribution of the frames. Point to point scheme as well as Contour scheme is used for the Implementation of this method. Aside from the number of subjects in each database, the main difference is that while the iris images are of very high quality, ear images are of somewhat lower quality. We have used Database of IIT Delhi for Ear Recognition \& Iris Database CASIA v2.

The verification rate of the standalone iris classifier is very high. The FAR and FRR rates at various thresholds are zero. It is difficult to compare and show the FAR and FRR rates using the ROC curve. Therefore, we simply calculated the total error rate (i.e., FAR+FRR). The outputs of RBF neural network are near 0 or 1 , so it is not appropriate to express it as FAR and FRR at every threshold.. It is apparent from the distribution of matching distances that fusion improves the separation of genuine scores from the impostor scores. As the ROC curves cannot be compared, we analyze the range of threshold values that result in high accuracy. The threshold values range that can be selected for high verification rates is larger for the fused matcher compared to the individual matchers.

Table 2: Feature vs FAR \& FRR Analysis

\begin{tabular}{|l|l|l|}
\hline Feature/ Analysis & FAR & FRR \\
\hline Iris & $99.2 \%$ & $0.8 \%$ \\
\hline Ear & $98.5 \%$ & $1.5 \%$ \\
\hline Fusion & $96.3 \%$ & $3.7 \%$ \\
\hline
\end{tabular}

\section{Conclusions}

The results obtained from the multimodal experiments suggest that the ear is a very suitable biometric as a noninvasive technique to be combined with other noninvasive techniques like face recognition, or gait. The novel approach using spatial segmentation for dividing the recognition problem and recognizing segments of the ear proved to be successful and yielded higher recognition rates than the base eigen-ear experiment. The segmentation technique is very useful for dealing with occlusions. The segmentation technique also gives flexibility to the system which makes it possible to recognize individuals with whatever segment of the ear that is available. The segmentation of the ear makes the calculations for the PCA faster since the images are divided to smaller images where the PCA calculations are done independently for every segment.

The approach of combining images post PCA calculations or in the PCA space for the multimodal experiments, so that each combination of images will be represented by two points instead of one point in the PCA space, makes the system even more flexible. The multimodality could be applied whenever the appropriate information from the biometrics involved is available or could be ignored and each system will work on its own without the need to have more than one implementation to accommodate each case. 


\section{References}

[1] Basit, A., Javed, M. Y. and Anjum, M. A., "Efficient iris recognition method for human identification", ENFORMATIKA, pp. 2426, vol 1, 2005.

[2] Moreno, B., Sanchez, A., Velez, J., F., "On the Use of Outer Ear Images for PersonalIdentification in Security Applications", IEEE 33rd Annual International Carnahan Conference on Security Technology, pp. 469-476, 1999.

[3] M. Ali, M. Y. Javed, A. Basit, 'Ear Recognition Using Wavelets', Proceedings of Image and Vision Computing New Zealand 2007, pp. 83-86, Hamilton, New Zealand, December 2007

[4] Cesar Santos, Edson J.R, Justino, Flavio Bertolozzi, Robert Sabaourin, "An offline Signature Verification Method Based on the questioned document expert's Approach and a Neural Network Classifier”, Catolica do Parana, Rua Imaculada onceicao,1155,Curitiba, PR, Brazil.

[5] John .G. Daugman, "How Iris Recognition Works”, IEEE Trans, Circuit and Syst for Video Tech., vol.14, no.1, pp213, Jan 2004 